\title{
Free energy landscape and kinetics of phase transition in two coupled SYK models and the corresponding wormhole-two black hole switching
}

\author{
Ran $\mathbf{L i}^{a, b}$ and Jin Wang ${ }^{b, c, 1}$ \\ ${ }^{a}$ School of Physics, Henan Normal University, \\ Xinxiang 45300\%, China \\ ${ }^{b}$ Department of Chemistry, Stony Brook University, \\ Stony Brook, NY 11794, U.S.A. \\ ${ }^{c}$ Department of Physics and Astronomy, Stony Brook University, \\ Stony Brook, NY 11794, U.S.A. \\ E-mail: liran@htu.edu.cn, jin.wang.1@stonybrook.edu
}

\begin{abstract}
We propose that the thermodynamics and the kinetics of the phase transition between wormhole and two black hole described by the two coupled SYK model can be investigated in terms of the stochastic dynamics on the underlying free energy landscape. We assume that the phase transition is a stochastic process under the thermal fluctuations. By quantifying the underlying free energy landscape, we study the phase diagram, the kinetic time and its fluctuations in details, which reveal the underlying thermodynamics and kinetics. It is shown that the first order phase transition between wormhole and two black hole described by two coupled SYK model is analogous to the Van der Waals phase transition. Therefore, the emergence of wormhole and two black hole phases, the phase transition and associated kinetics can be quantitatively addressed in our free energy landscape and kinetic framework through the dependence on the barrier height and the temperature.
\end{abstract}

KeYwORDS: Random Systems, 2D Gravity, Gauge-gravity correspondence

ArXiv EPrint: 2109.07635

\footnotetext{
${ }^{1}$ Corresponding author.
} 


\section{Contents}

1 Introduction 1

2 Two coupled SYK model 2

$3 \quad$ Free energy landscape $\quad 4$

4 Kinetics of phase transition on the free energy landscape 10

$\begin{array}{lll}4.1 & \text { Fokker-Planck equation } & 10\end{array}$

4.2 Analytical results of the kinetic time and its fluctuation 11

4.3 Numerical results of the kinetic time and its fluctuation 13

5 Conclusion $\quad 16$

\section{Introduction}

Originally, wormholes are the imaginary objects that are used for time travels or fastthan-light journeys in science fictions. However, it is shown that wormholes [1-4] as the special spacetime structures play more and more important role in understanding the physics of our universe. Recent examples include: the Maldacena and Susskind's proposal of "ER=EPR" conjecture [5], the traversable wormholes from the double trace coupling between the boundaries of AdS black hole [6], Hayden-Preskill protocol realization [7] or quantum teleportation towards traversable wormholes [8], replica wormholes used to derive the island rule for the entanglement entropy of Hawking radiation [9-11], and humanly traversable wormhole solutions in general relativity [12, 13]. Wormholes have attracted significant research attentions in recent years.

Traversable wormholes constructed by turning on the interaction that couples the two boundaries of an eternal AdS black hole [6] is an important example that may have an interesting interpretation in the context of $\mathrm{ER}=\mathrm{EPR}$ conjecture. In particular, Maldacena and Qi [14] have constructed the nearly- $A d S_{2}$ solution [15-18] describing an eternal traversable wormhole by introducing the boundary interaction in Jackiw-Teitelboim (JT) gravity $[19,20]$. It is also shown that the system undergoes a first order transition at finite temperature from the wormhole phase to a two black holes phase, which displays the usual Hawking-Page phase transition [21] between the thermal AdS phase at low temperature and the black hole phase at high temperature. They further argued that, in the dual two Sachdev-Ye-Kitaev (SYK) systems [22-25] coupled by a relevant interaction, there is a similar phase diagram. The following analytical and numerical calculations confirm this argument [26-31].

In the present work, for the two coupled SYK model at the large $N$ limit, according to the thermodynamics of the system, we define the off-shell free energy and quantify the 
corresponding free energy landscape. In the framework of the free energy landscape [32-35], we analyze the phase diagram between the wormhole and two black hole described by the two coupled SYK model and obtain the conclusion that the phase transition in this model is analogous to the Van der Waals type phase transition [36-38], not exactly the HawkingPage phase transition [39]. Furthermore, we propose the kinetics of the phase transition between the wormhole and two black hole can be investigated by using the stochastic dynamics on the free energy landscape. We assume the phase transition between the wormhole and two black hole is a stochastic process under the thermal fluctuations and study the probabilistic Fokker-Planck equation which describes the kinetics of the phase transition [38-49]. By calculating the kinetics time and its fluctuation, we reveal the underlying thermodynamics and kinetics of the phase transition between the wormhole and two black hole. It is shown that the underlying reason of the kinetic behavior is determined by the barrier heights and the temperature on the free energy landscape.

We emphasize that the first order phase transition between the wormhole and two black hole in two coupled SYK model is not Hawking-Page type phase transition [39]. Our argument is based on the following three points. The shape of the free energy landscape is double well, which is different from the shape of the Hawking-Page transition [39]. However, one of the double well here locates at the position where the order parameter is very close to zero. This is very similar to the free energy landscape of the Hawking-Page transition where one of the well is at zero. In this sense, both Hawking-Page type landscape and double wells describe first order phase transition. The second point is the existence of the critical point as in the gas-liquid phase transition in van der waals fluid [36-38]. The third point is that the critical exponents near the critical point coincide with those of Van der Waals fluid. Therefore, we conclude the first order phase transition in two coupled SYK model is analogous to the Van der Waals phase transition rather than Hawking-Page type.

The rest of the paper is arranged as follows. In section 2, we review the basic fact about the two coupled SYK model and its thermodynamics at the large $N$ limit. In section 3 , we define the off-shell free energy and quantify the free energy landscape. Using the free energy landscape topography, we analyse the phase diagram between the wormhole and two black hole of the system. In section 4, using the Fokker-Planck equation, we calculate the kinetic time and its fluctuation as the functions for the phase transition of the temperature and study the underlying reason. The summary and discussion are presented in the last section.

\section{Two coupled SYK model}

The SYK model has a Hilbert space generated by $N$ Majorana fermions $\psi^{i}$ with a Hamiltonian of the form $[22,23]$

$$
\begin{aligned}
H & =(i)^{q / 2} \sum_{1 \leq j_{1} \leq j_{2} \cdots \leq j_{q}} J_{j_{1} j_{2} \cdots j_{q}} \psi^{j_{1}} \psi^{j_{2}} \cdots \psi^{j_{q}}, \\
\left\langle J_{j_{1} \cdots j_{q}}^{2}\right\rangle & =\frac{2^{q-1} \mathcal{J}^{2}(q-1) !}{q N^{q-1}} \quad \text { (no sum) }
\end{aligned}
$$


where the couplings are drawn from a random gaussian distribution with the mean indicated above. The factor of $i$ is necessary to maintain a hermitian Hamiltonian when $q / 2$ is odd.

The Hamiltonian of the two coupled SYK model is given by [14]

$$
H_{\text {total }}=H_{\mathrm{L}, \mathrm{SYK}}+H_{\mathrm{R}, \mathrm{SYK}}+H_{\mathrm{int}}, \quad H_{\mathrm{int}}=i \mu \sum_{j} \psi_{L}^{j} \psi_{R}^{j}
$$

where $H_{\mathrm{L}, \mathrm{SYK}}$ and $H_{\mathrm{R}, \mathrm{SYK}}$ are the Hamiltonians of the two decoupled SYK models and $H_{\text {int }}$ denotes the interaction of the two copies of the SYK model with $\mu$ being the coupling constant. We denote the fermions of the two copies the SYK model as $\psi_{L}^{i}$ and $\psi_{R}^{i}$. For the decoupled models, $H_{\mathrm{L}, \mathrm{SYK}}$ and $H_{\mathrm{R}, \mathrm{SYK}}$ are described by the same couplings, up to a sign for odd $q / 2, J_{j_{1} \cdots j_{q}}^{L}=(-1)^{q / 2} J_{j_{1} \cdots j_{q}}^{R}$.

We consider the large $N$ case. The effective action can be written as [14]

$$
\begin{aligned}
-S_{E} / N= & \log \operatorname{Pf}\left(\partial_{\tau} \delta_{a b}-\Sigma_{a b}\right)-\frac{1}{2} \int d \tau_{1} d \tau_{2} \sum_{a, b}\left[\Sigma_{a b}\left(\tau_{1}, \tau_{2}\right) G_{a b}\left(\tau_{1}, \tau_{2}\right)-s_{a b} \frac{\mathcal{J}^{2}}{2 q^{2}}\left[2 G_{a b}\left(\tau_{1}, \tau_{2}\right)\right]^{q}\right]+ \\
& +\frac{i \mu}{2} \int d \tau_{1}\left[-G_{L R}\left(\tau_{1}, \tau_{1}\right)+G_{R L}\left(\tau_{1}, \tau_{1}\right)\right] .
\end{aligned}
$$

Here $a, b=L, R$ denotes the two sides. Note that the functions obey the antisymmetry condition $G_{a b}\left(\tau_{1}, \tau_{2}\right)=-G_{b a}\left(\tau_{2}, \tau_{1}\right)$. Here $s_{a b}$ denotes a sign, $s_{L L}=s_{R R}=1, s_{L R}=$ $s_{R L}=(-1)^{q / 2}$, which arises because for odd $q / 2$ the signs of the couplings of the left Hamiltonian are the opposite compared to those of the right Hamiltonian (with the same absolute value). The Green's functions satisfy the Schwinger-Dyson equations [14], which are not listed for simplicity.

In the large $q$ limit, the effective action is amenable to an analytical calculation [14]. When the inverse temperature $\beta$ is of order $q \log q$, the logarithm of the partition function $\log Z / N$ is given by [14]

$$
\frac{\log Z}{N}=\frac{\tanh \tilde{\gamma} \log (q / \sigma)}{q}\left[\frac{q}{2}-1+\frac{1}{\tanh \gamma \tanh \tilde{\gamma}}+\log \frac{\sinh \gamma}{\cosh \tilde{\gamma}}+\frac{\sigma}{\tanh \tilde{\gamma}}\right]+\frac{\sigma}{q},
$$

where the parameters are defined as

$$
\begin{array}{rlrl}
\sigma=q e^{-\beta \nu}, & \nu=\frac{2 \alpha}{q}=\frac{\mu}{\tanh \tilde{\gamma}}, & \alpha & =\mathcal{J} \sinh \gamma, \\
\tilde{\gamma}=\gamma+\sigma, & \hat{\mu}=\mu q=2 \alpha \tanh \tilde{\gamma}, & \log (q / \sigma)=\frac{\beta \mu}{\tanh \tilde{\gamma}} .
\end{array}
$$

It should be noted that eq. (2.5) determine the inverse temperature $\beta$ and the parameters $\alpha$, and $\gamma$ in the solution as the functions of the parameter $\sigma$. However, it turns out that the system temperature $T_{s}(\sigma)=\beta^{-1}(\sigma)$ is not a monotonic function of $\sigma$. Due to the non-monotonicity of $T_{s}$, there is a temperature window $T_{1}<T_{s}<T_{2}$ in which there are three solutions with different energy $E$ for a given temperature. They correspond to three saddle points of the free energy, including two minima and one saddle point. This point will be explicitly demonstrated on the free energy landscape in the next section. 


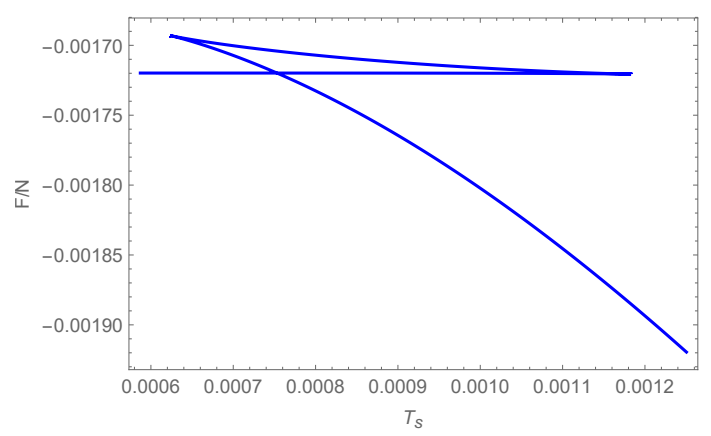

Figure 1. On-shell free energy $\tilde{F}_{\text {on-shell }}=\frac{F}{N}$ as a function of the temperature $T_{s}$ of the system. In this plot, $q=50, \mathcal{J}=1$, and $\hat{\mu}=0.1$. There is a first order phase transition at the temperature $T_{s}=0.000753$.

From eq. (2.4), one can compute the free energy, the energy and also the entropy of the system, which are given by [14]

$$
\begin{aligned}
\tilde{F}_{\text {on-shell }} & \equiv \frac{F}{N}=-\frac{\hat{\mu}}{q^{2}}\left[\frac{q}{2}-1+\frac{1}{\tanh \gamma \tanh \tilde{\gamma}}+\log \frac{\sinh \gamma}{\cosh \tilde{\gamma}}+\frac{\sigma}{\tanh \tilde{\gamma}}+\frac{\sigma}{\tanh \tilde{\gamma} \log (q / \sigma)}\right] \\
\tilde{E} & \equiv \frac{E}{N}=\frac{\hat{\mu}}{q^{2}}\left[-\frac{q}{2}+1-\frac{1}{\tanh \gamma \tanh \tilde{\gamma}}-\log \frac{\sinh \gamma}{\cosh \tilde{\gamma}}\right] \\
\tilde{S} & \equiv \frac{S}{N}=\frac{\sigma}{q}\left[1+\log \frac{q}{\sigma}\right] .
\end{aligned}
$$

Where we have denote the free energy calculated from the partition function as the on-shell free energy $\tilde{F}_{\text {on-shell. }}$.

The on-shell free energy of the two coupled SYK model as the function of the system temperature $T_{s}$ is plotted in figure 1 . When $0.000623<T_{s}<0.00118$, there are three phases [14], which are the stable wormhole phase, the unstable wormhole phase and the two black hole phase as depicted in figure 2. The stable phase is the one that has the lowest on-shell free energy.

\section{$3 \quad$ Free energy landscape}

We are interested in the emergence of wormhole and two black hole phases and the corresponding first order phase transition on the free energy landscape. In the formalism of the free energy landscape [32-35], the Gibbs free energy is defined as the continuous function of the order parameter or reaction coordinate of the system. The order parameter or reaction coordinate may be considered as the coarse-grained description which captures the essential characteristics and the microscopic degree of freedom of the system [38-49].

Let us firstly consider the system temperature $T_{s}=1 / \beta$ as the function of the parameter $\sigma$. In figure 3 , it is shown that the system temperature $T_{s}$ is not a monotonic function of $\sigma$. This is similar to the relationships between the temperature and black hole radius for the Hawking-Page transition [39] or the small/big RNAdS black hole phase transition [38], where the black hole radii are treated as the order parameters. Therefore, the 


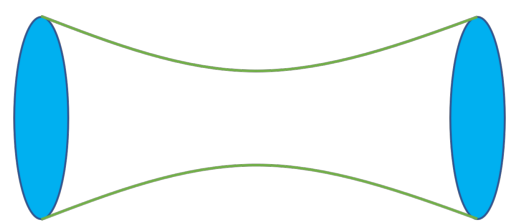

(a)
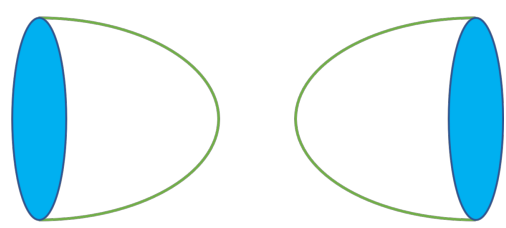

(b)

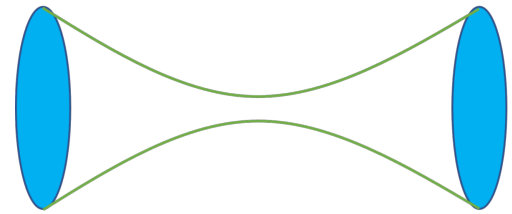

(c)

Figure 2. (a) Stable wormhole phase in the low temperature; (b) two black hole phase in the high temperature; (c) unstable wormhole phase in the low temperature. Note that these pictorial representations are in the euclidean signature.

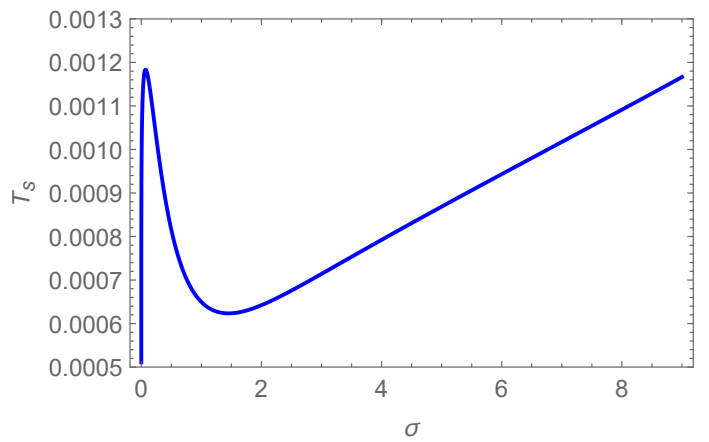

Figure 3. The system temperature $T_{s}$ as the function of the parameter $\sigma$. The non-monotonicity is shown explicitly. In this plot, $q=50, \mathcal{J}=1$, and $\hat{\mu}=0.1$.

non-monotonic relationship between the temperature and the parameter $\sigma$ prompts us the possibility of selecting $\sigma$ as the order parameter.

Then we also consider the relationship between the coupling constant $\hat{\mu}$ and the parameter $\sigma$ in figure 4. It is shown that their functional relationship is also non-monotonic. However, when $\sigma$ approaches zero, the coupling constant $\hat{\mu}$ becomes large, and when $\sigma$ becomes large, $\hat{\mu}$ approaches zero. Therefore, $\sigma$ represents the coupling strength of the two SYK models to a certain degree. In fact, the emergence of the three phases (the stable wormhole phase, the unstable wormhole phase and the two black hole phase) in figure 2 should reflect the coupling strength of the two coupled SYK models [14]. This observation complemented with the non-monotonic relationship between $T_{s}$ and $\sigma$ indicates that the parameter $\sigma$ can be selected to be the order parameter. 


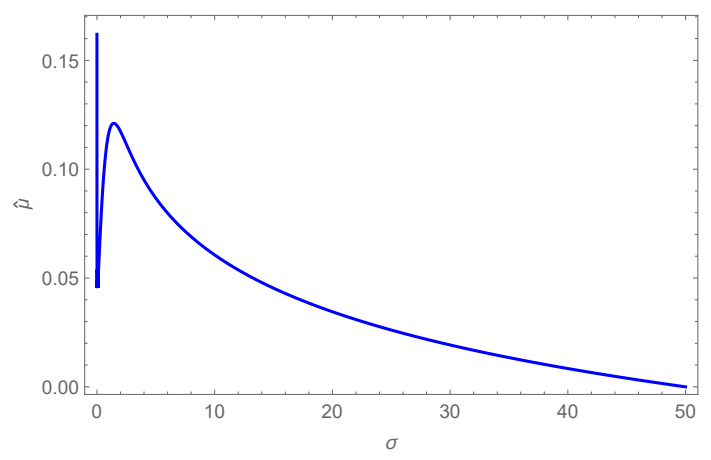

Figure 4. The coupling constant $\mu$ as the function of the parameter $\sigma$. The non-monotonicity is shown explicitly. In this plot, $q=50, \mathcal{J}=1$, and $T_{s}=0.000753$.

Note that the selection of the order parameter is not unique. As proposed in refs. [38$40,42,46]$, for the landscape descriptions of black hole phase transitions, the order parameter was selected to be the radius of the fluctuating black hole. It was also proposed in [50] that the number density of the black hole molecules can be chosen as the order parameter to measure the microscopic degrees of freedom of the black hole from the viewpoint of thermodynamic phase transition. We believe that the discussion based on these proposals will give quantitatively the similar results. Another point is about the implication of the order parameter in the context of black hole and wormhole. Because SYK model is the dual description of the nearly $A d S_{2}$ gravity, the order parameter can be treated as the coarse-grained description emergent from the microscopic degrees of freedom of the black hole and wormhole spacetime.

We select $\sigma$ as the order parameter of the system. $\sigma$ should be considered as the independent variable. We consider the canonical ensemble at the specific temperature $T$. The temperature $T$ should be considered as the ensemble temperature, and is not determined by the order parameter $\sigma$. In order to construct the free energy landscape, we need to specify every state in the ensemble a Gibbs free energy. In other words, we should define the off-shell free energy as a function of the order parameter and the ensemble temperature. Recall that the on-shell free energy is given by the thermodynamic relationship $F=E-T_{s} S$, where $T_{s}$ is the temperature of the system, which is different from the ensemble temperature $T$. Now we generalized this definition to the off-shell free energy as

$$
\begin{aligned}
\tilde{F} & =\tilde{E}-T \tilde{S} \\
& =\frac{\hat{\mu}}{q^{2}}\left[-\frac{q}{2}+1-\frac{1}{\tanh \gamma \tanh \tilde{\gamma}}-\log \frac{\sinh \gamma}{\cosh \tilde{\gamma}}\right]-\frac{\sigma}{q}\left[1+\log \frac{q}{\sigma}\right] T
\end{aligned}
$$

where $T$ denotes the ensemble temperature. Without special remark, $T$ denotes the ensemble temperature but not the temperature of the system in the following. In the above expression, $\tilde{\gamma}$ and $\gamma$ are considered as the functions of $\sigma$ by the following relations

$$
\tilde{\gamma}=\gamma+\sigma, \quad \hat{\mu}=2 \mathcal{J} \sinh \gamma \tanh \tilde{\gamma} .
$$

The definitions of the order parameter and the off-shell free energy is the generalization of the definitions for the black hole systems $[51,52]$. The similar consideration has been 


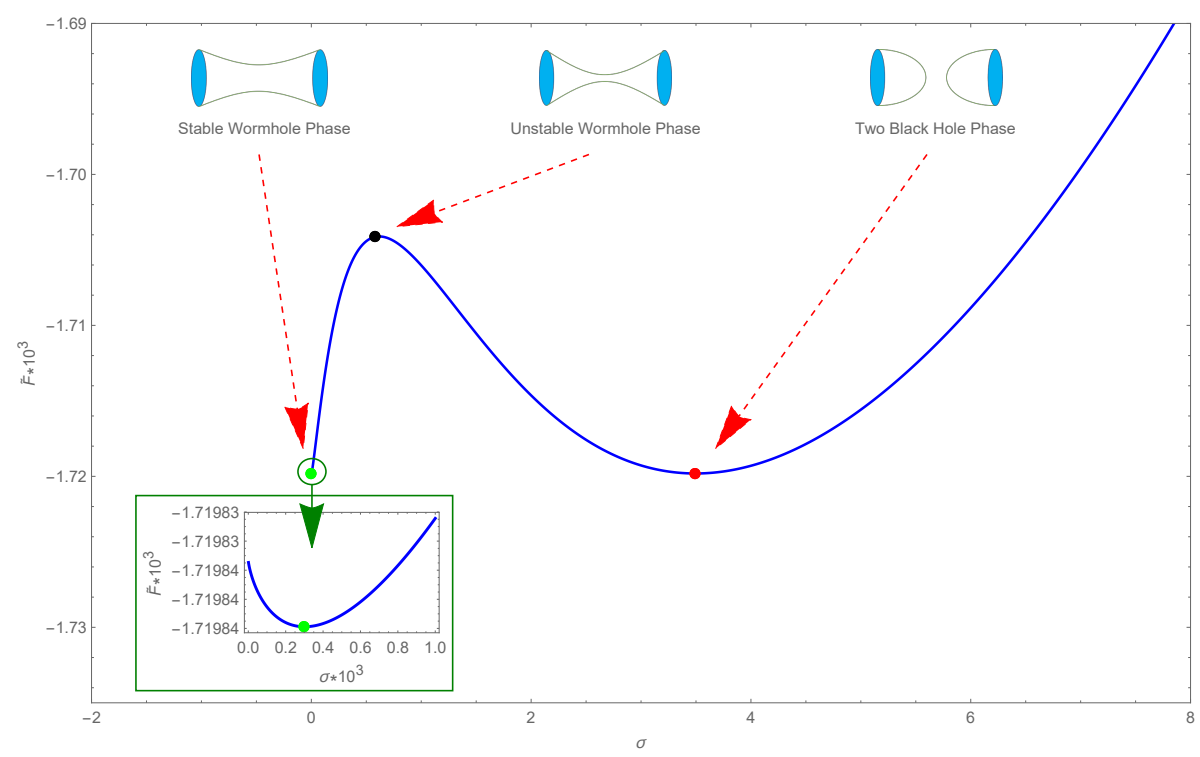

Figure 5. Free energy landscape, i.e. the off-shell free energy as the function of the order parameter $\sigma$. The sub-figure shows the potential well near $\sigma=0$. It is shown that the landscape has the shape of the double well and three saddle points representing the wormhole phases and two black hole phase. In this plot, $q=50, \mathcal{J}=1, \hat{\mu}=0.1$, and $T=0.000753$.

applied to study the black hole phase transition, for example, the Hawking-page phase transition [38], the small/large RNAdS black hole phase transition [39], and the phase transition of the Schwarzschild black hole in cavity [53, 54]. As explained, $\sigma$ is selected to be the order parameter. In the definition of the off-shell free energy, $\sigma$ is then considered to be independent variable, and the temperature is defined as the ensemble temperature and is kept fixed during the kinetic process, in analogy to the statistical physics of canonical ensemble. In general, there can be multiple paths between the minima of the free energy landscape. However, different paths have different weights. Here, we select the most probable path with the highest weight which connects the saddle point on the free energy landscape between the free energy landscape basins and study the corresponding kinetics. Since the weight of the path is related exponentially to the action, the other paths with smaller weights are exponentially suppressed due to the differences in actions [55]. Therefore, one can focus on the most probable or dominant path and approximately ignore the other sub-leading paths and their contributions to the weights.

The free energy landscape, which was a visualized image of the functional relation between the off-shell free energy and the order parameter, is plotted in figure 5. The subfigure shows the potential well near $\sigma=0$. It is shown that the free energy landscape has the shape of the double well. This is different from the single well of Hawking-Page transition [38] but similar to the double well shape of the free energy landscape of the small/large RNAdS black hole phase transition [39]. Therefore, the first order phase transition should be van der Waals type. The three saddle points are displayed by the red, the green and the black dots correspondingly. According to the discussion above, the red, the green and the black dots on the free energy landscape corresponds to two black hole phase, stable worm- 

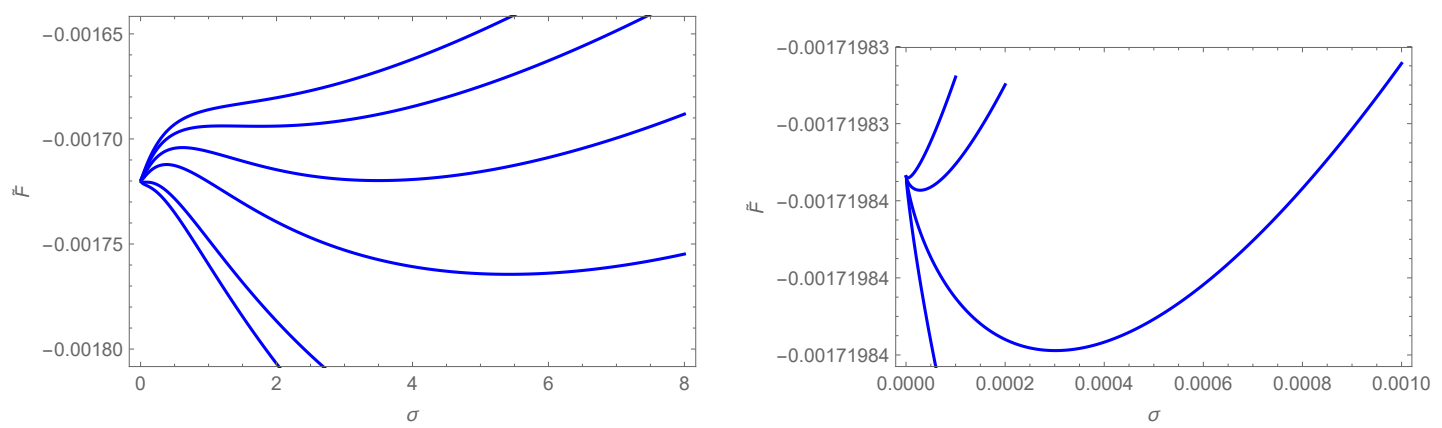

Figure 6. Free energy landscape at different ensemble temperatures. The right panel is the zoom in picture of the left panel. In the left panel, the ensemble temperatures are $0.00055,0.00063,0.000753,0.0009,0.00118$, and 0.0013 from the top to bottom. In the right panel, the ensemble temperatures are $0.00055,0.00063,0.000753$, and 0.0009 from the top to bottom. In this plot, $q=50, \mathcal{J}=1$, and $\hat{\mu}=0.1$.

hole phase and unstable wormhole phase respectively. For latter convenience, we denote the order parameters of the three phases as $\sigma_{s}$ for the stable wormhole phase, $\sigma_{m}$ for the unstable wormhole phase, and $\sigma_{l}$ for the two black hole phase. The ensemble temperature for the plots in figure 5 is taken as the phase transition temperature $T_{\mathrm{ph}}=0.000753$, in which case the left and right wells representing the stable wormhole phase and the two black hole phase have the same depths.

From the free energy landscape plotted in figure 5 and the corresponding labels, one can observe that the order parameter $\sigma$ is related to the inverse of the size of the wormhole throat. It is also shown in [14] that when the parameter $\sigma$ becomes larger, the left-right Lorentzian correlator decreases. A small $\sigma$ implies a large size of the wormhole throat and a large left-right correlator, giving rise to a stable traversable wormhole that can transfer information from one side to the other. An intermediate $\sigma$ implies that the wormhole throat is shrinking, or at least is not as "transparent" and easy to cross as it was at the small $\sigma$, giving rise to connected yet unstable wormhole. A large $\sigma$ implies that the left-right correlator is very small, and the wormhole is closing and non-transparent, giving rise to separate two black hole phase.

In figure 6 , we show the free energy landscapes at different ensemble temperatures. For $\hat{\mu}=0.1$, there are two temperatures $T_{1}=0.00063$ and $T_{2}=0.00118$. We find that when $T_{1}<T<T_{2}$, three phases (two stable and one unstable phases) coexist and the free energy landscape has the shape of double well. In particular, when $T_{1}<T<T_{\mathrm{ph}}$, the depth of the left well representing the stable wormhole phase is lower than the depth of the right well representing the two black hole phase, which implies that the wormhole phase is thermodynamically favored. When $T_{\mathrm{ph}}<T<T_{2}$, the conclusion is exchanged and the two black hole phase is favored. When $T<T_{1}$ or $T>T_{2}$, the free energy landscape has the shape of the single well. From figure 6, we also find that the depth of the right well changes significantly when varying the temperature, while the depth of the left well is insensitive.

In order to confirm the argument of the phase transition type, we further calculate the phase diagram of the system, which is presented in figure 7 . The stable wormhole 


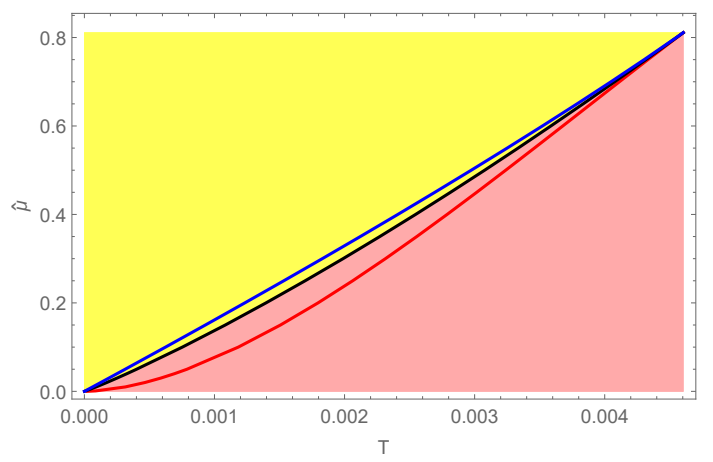

Figure 7. Phase diagram in $\hat{\mu}-T$ plane. In this plot, $q=50$ and $\mathcal{J}=1$.

phase is thermodynamically favored in the yellow region, while the two black hole phase is thermodynamically favored in the pink region. For specific coupling constant $\hat{\mu}$, there is a temperature window $T_{1}<T<T_{2}$ in which three phases coexist and the free energy landscape has the shape of double well. Varying the parameter $\hat{\mu}$, we can get $T_{1}$ and $T_{2}$ as the function of $\hat{\mu}$. These two curves $T_{1}(\hat{\mu})$ and $T_{2}(\hat{\mu})$ are plotted in blue and red in figure 7 . There is also a phase transition temperature, which is plotted in black. The phase transition temperature is determined by equal free energies of the two basins. The black line represents the coexistence curve that the stable wormhole phase and the two black hole phase coexist in equal probability. When $\hat{\mu} \rightarrow 0$ or $\hat{\mu}$ increases, the temperature window closes. It is shown there is a critical point as in the gas-liquid phase transition of van der Waals fluid. At the critical point, $\hat{\mu}=0.811$, and $T=0.0046$. This behavior is analogous to the van der Waal type phase transition in thermodynamics.

Now we calculate the critical exponent near the critical point. Note that the critical point has been revealed from the $\hat{\mu}-T$ phase diagram. It is natural to consider $\hat{\mu}$ as the 'pressure' of the van der Waals system. The equation (2.5) can be treated as the equation of state relating the three state parameters, i.e., the 'pressure' $\hat{\mu}$, the order parameter $\sigma$, and the temperature $T_{s}$. Firstly, we calculate the critical point numerically. The critical point satisfies the equations $\left.\frac{\partial \hat{\mu}}{\partial \sigma}\right|_{T_{s}}=\left.\frac{\partial^{2} \hat{\mu}}{\partial \sigma^{2}}\right|_{T_{s}}=0$. For $q=50$ and $\mathcal{J}=1$, the numerical results give $\hat{\mu}_{c}=0.883$ and $T_{c}=0.00493$. This is consistent with the results obtained from studying the free energy landscape within the range of the numerical error.

The existence of the critical point implies that the Taylor expansion around the critical point must start with the cubic term

$$
\hat{\mu}-\hat{\mu}_{c} \sim\left(\sigma-\sigma_{c}\right)^{3},
$$

where $\sigma_{c}$ is the critical value of the order parameter. The exponent that govern the change of the order parameter along with the 'pressure' $\hat{\mu}$ on the critical isotherm $T=T_{c}$ is 3 .

Then, we consider the exponent that determines the behavior of the isothermal compressibility

$$
\kappa_{T}=-\left.\frac{1}{\sigma} \frac{\partial \sigma}{\partial \hat{\mu}}\right|_{T}
$$


Because at the critical point $\left.\frac{\partial \hat{\mu}}{\partial \sigma}\right|_{T_{s}}=0$, expanding for temperatures close to $T_{c}$, we expect

$$
\left.\frac{\partial \hat{\mu}}{\partial \sigma}\right|_{T, \sigma_{c}} \sim\left(T-T_{c}\right) .
$$

This indicates that the compressibility should diverge at the critical point, with the scaling as

$$
\kappa_{T} \sim\left(T-T_{c}\right)^{-1}
$$

which gives the exponent that determines the behavior of the isothermal compressibility as being 1 .

Next, we consider that the exponent governs the behaviour of the specific heat at constant order parameter

$$
C_{\sigma}=\left.T \frac{\partial S}{\partial T}\right|_{\sigma}
$$

Note that in eq. (2.6), the entropy is independent of $T$ if taking $\sigma$ as the independent parameter. Therefore, this exponent is 0 .

At last, we consider that the exponent describes the behaviour of the order parameter $\epsilon=\sigma_{\mathrm{BH}}-\sigma_{\mathrm{WH}}$ (the difference of the order parameters of the two black hole phase and the wormhole phase) on the given isotherm. For $T<T_{c}$, the state equation has two stable solutions. Then, from eq. (2.5), we have

$$
T=\frac{2 \mathcal{J}}{q} \frac{\sinh \gamma}{\log (q / \sigma)} .
$$

Close to the critical point, we can write $\sigma_{\mathrm{BH} / \mathrm{WH}}=\sigma_{c} \pm \epsilon / 2$ and $\gamma_{\mathrm{BH} / \mathrm{WH}}=\gamma_{c} \pm \delta / 2$. Substituting these into the following equation

$$
\frac{\sinh \gamma_{\mathrm{BH}}}{\log \left(q / \sigma_{\mathrm{BH}}\right)}=\frac{\sinh \gamma_{\mathrm{WH}}}{\log \left(q / \sigma_{\mathrm{WH}}\right)},
$$

one can get $\epsilon \sim \delta$. Then, expanding eq. (3.8) near the critical point and keeping just the leading order term, we get

$$
T-T_{c} \sim \epsilon^{2} \Rightarrow \epsilon \sim\left(T-T_{c}\right)^{1 / 2} .
$$

The exponent that describes the behaviour of the order parameter is $\frac{1}{2}$. The results of the exponents coincide with those of the Van der Waals system. This further supports the conclusion that the type of the phase transition is the van der Waals type.

\section{Kinetics of phase transition on the free energy landscape}

\subsection{Fokker-Planck equation}

We discuss the effective theory of the stochastic dynamics of the phase transition. Note that the generation of the fluctuating black hole is completely stochastic and the dynamical process is assumed to have the coarse-grained description by using the order parameter. In this section, we will study the kinetics of the phase transition based on the free energy 
landscape. As shown in the last section, we know that the free energy landscape as a function of the order parameter $\sigma$ exhibits double basin shape when the temperature lies in the region $\left[T_{1}, T_{2}\right]$.

We consider the case where are a large number of states in a thermodynamic ensemble in which one or a group of them can represent the wormholes or the two black hole phases during the state transition process. The probability distribution of the states (on-shell solutions as well as the off-shell solutions on the free energy landscape) are denoted by $\rho(\sigma)$. The stochastic kinetics of states under the thermal fluctuation can be described by the probabilistic Fokker-Planck equation, which on the free energy landscape is explicitly given by [38-49]

$$
\frac{\partial \rho(\sigma, t)}{\partial t}=D \frac{\partial}{\partial \sigma}\left\{e^{-\tilde{\beta} \tilde{F}(\sigma)} \frac{\partial}{\partial \sigma}\left[e^{\tilde{\beta} \tilde{F}(\sigma)} \rho(\sigma, t)\right]\right\}
$$

In the above equation, the diffusion coefficient $D$ is given by $D=k T / \zeta$ with $k$ being the Boltzman constant and $\zeta$ being dissipation coefficient. Without the loss of generality, we will take $k=\zeta=1$ in the following. Note that $\tilde{\beta}=1 / k T$ is the inverse ensemble temperature. Note that $\tilde{F}(\sigma)$ is the off-shell free energy as a function of the order parameter $\sigma$ modulated by the ensemble temperature $T$.

We have assumed that the order parameter emerges from certain combination of the microscopic degrees of freedom while the rest of the microscopic degrees of freedom plays the role of an effective heat bath interacting with the order parameter. Note that the dissipation coefficient $\zeta$ denotes the strength of the interaction between the order parameter and the effective thermal bath. These assumptions have been discussed in detail in [40]. In fact, the underlying dynamical equation is given by the Langevin equation that describes the dynamics of the order parameter. For different interaction strength, the corresponding evolution equation of the probability of the state in the ensemble is different. However, without the loss of generality, we consider the Langevin equation in the overdamped regime.

\subsection{Analytical results of the kinetic time and its fluctuation}

We now study the kinetics through the mean first passage time for the state switching process. Our purpose is to find out the time that it takes for a state starting from one local (global) stable phase and ending to another global (local) stable phase. Problems of this sort can be solved by the first passage time. In general, first passage time is defined as the time required for a state from the local (global) stable phase (either stable wormhole or two black hole described by the local (global) minimum of Gibbs free energy) to reach the transition state (an unstable intermediate wormhole phase determined by the maximum of Gibbs free energy or barrier in the present case) for the first time. Since we are considering a stochastic process under thermal fluctuation, the first passage time is a random variable. Thus, we are interested in the mean first passage time particularly. The mean first passage time quantifies an average timescale for a stochastic event of switching to first occur.

According to the discussion in [39], one can obtain the analytical integration expression for the mean first passage time from the stable wormhole phase to the unstable wormhole 
phase as

$$
\langle t\rangle=\frac{1}{D} \int_{\sigma_{s}}^{\sigma_{m}} d \sigma \int_{0}^{\sigma} d \sigma^{\prime} e^{\tilde{\beta}\left(\tilde{F}(\sigma)-\tilde{F}\left(\sigma^{\prime}\right)\right)} .
$$

Note that $\sigma_{s}$ and $\sigma_{m}$ are the order parameters for the stable wormhole phase and the unstable wormhole phase. In deriving this expression, we have imposed the reflecting boundary at $\sigma=0$.

The analytical integral expressions for the mean first passage time from the two black hole phase to the unstable wormhole phase can also be derived similarly, which is given as follows

$$
\langle t\rangle=\frac{1}{D} \int_{\sigma_{m}}^{\sigma_{l}} d \sigma \int_{\sigma}^{+\infty} d \sigma^{\prime} e^{\tilde{\beta}\left(\tilde{F}(\sigma)-\tilde{F}\left(\sigma^{\prime}\right)\right)} .
$$

Note that $\sigma_{l}$ denotes the order parameter of the two black hole phase. With these expressions, we can compute mean first passage time via numerical integration directly without concern on the time distribution.

It is shown in [40] that by performing the Gaussian integral, the approximate expression of the mean first passage time can be obtained. For the transition process from the stable wormhole phase to the unstable wormhole phase, it is given by

$$
\langle t\rangle \approx \frac{\pi \zeta}{\omega_{s} \omega_{m}} e^{\beta\left(\tilde{F}\left(\sigma_{m}\right)-\tilde{F}\left(\sigma_{s}\right)\right)},
$$

where $\omega_{s}$ and $\omega_{m}$ are the oscillating frequencies of the stable wormhole phase and the unstable wormhole phase on the free energy landscape. A similar expression can be derived for the transition process from the two black hole phase to the unstable wormhole phase.

We can see that the transition times between the wormhole phase and the two black hole phase in both directions are determined by the exponential factor and prefactor. The most sensitive dependence of the switching rate is on the exponential. Two elements determine the trend, free energy barrier height and temperature. The transition rate is significantly increased as the free energy barrier over temperature between the wormhole phase and two black hole phase increases. Note that the free energy landscape and the associated barrier are dependent on the temperature. Therefore, when temperature changes, the free energy barrier also changes accordingly. For fixed temperature, the switching rate is significantly increased as the free energy barrier between the wormhole phase and two black hole phase increases. For fixed barrier height, the switching rate is significantly increased as the temperature increases. Therefore, both the free energy landscape topography quantified by the barrier height and temperature can determine how difficult it is to switch from wormhole phase to the two black hole phase or vice versa.

In the black hole/wormhole context, the higher interaction strength indicates stronger coupling among the microscopic degrees of freedom of the black hole/wormhole spacetime. Strong coupling imposes strong constraints to the system. As a result of the stronger interactions/couplings, the state switching and phase transition between the black hole and wormhole are kinetically harder to realize in the over-damped regime considered in the current study. This can be seen clearly seen from the approximate expression eq. (4.4) of the kinetic time of the phase transition versus friction coefficient. 

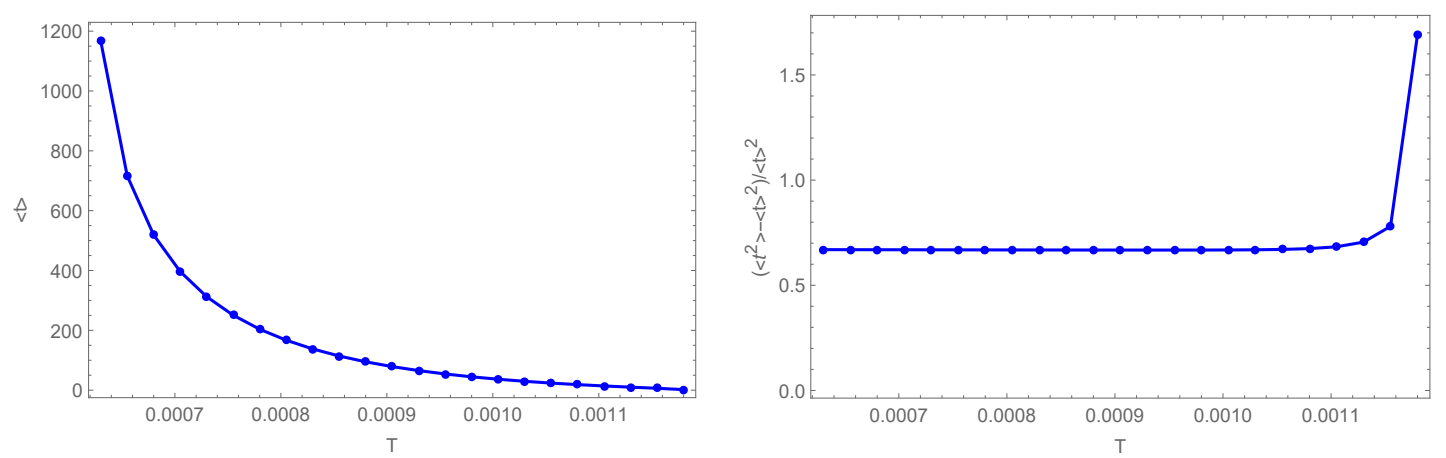

Figure 8. Mean first passage time (left panel) and the relative fluctuation (right panel) of the transition process from the stable wormhole phase to the unstable wormhole phase. In this plot, $q=50, \mathcal{J}=1$, and $\hat{\mu}=0.1$.

We can also derive the following analytical integration expression of $\left\langle t^{2}\right\rangle$ for the state transition process from the stable wormhole phase to the unstable wormhole phase that can be used to compute the kinetic fluctuations of the first passage time

$$
\left\langle t^{2}\right\rangle=\frac{2}{D^{2}} \int_{\sigma_{s}}^{\sigma_{m}} d \sigma \int_{0}^{\sigma} d \sigma^{\prime} \int_{\sigma^{\prime}}^{\sigma_{m}} d r^{\prime \prime} \int_{0}^{\sigma^{\prime \prime}} d \sigma^{\prime \prime \prime} e^{\tilde{\beta}\left(\tilde{F}(\sigma)-\tilde{F}\left(\sigma^{\prime}\right)+\tilde{F}\left(\sigma^{\prime \prime}\right)-\tilde{F}\left(\sigma^{\prime \prime \prime}\right)\right)},
$$

and the similar analytical integral expressions of $\left\langle t^{2}\right\rangle$ for the state transition process from the two black hole phase to the unstable wormhole phase

$$
\left\langle t^{2}\right\rangle=\frac{2}{D^{2}} \int_{\sigma_{m}}^{\sigma_{l}} d \sigma \int_{\sigma}^{+\infty} d \sigma^{\prime} \int_{\sigma_{m}}^{\sigma^{\prime}} d \sigma^{\prime \prime} \int_{\sigma^{\prime \prime}}^{+\infty} d \sigma^{\prime \prime \prime} e^{\tilde{\beta}\left(\tilde{F}(\sigma)-\tilde{F}\left(\sigma^{\prime}\right)+\tilde{F}\left(\sigma^{\prime \prime}\right)-\tilde{F}\left(\sigma^{\prime \prime \prime}\right)\right)} .
$$

\subsection{Numerical results of the kinetic time and its fluctuation}

Firstly, we consider the kinetics and its fluctuation of the transition process from the stable wormhole phase (the green dot on the free energy landscape in figure 5) to the unstable wormhole phase (black dot on the free energy landscape). We have assumed that this switching process dominates the phase transition process from the stable wormhole phase to the two black hole phase.

In general, the kinetics of the transition process can be described by two quantities, i.e. the mean first passage time and its fluctuation. Here, we mainly consider the relative fluctuation, which is defined as $\left(\left\langle t^{2}\right\rangle-\langle t\rangle^{2}\right) /\left\langle t^{2}\right\rangle$. According to the analytical integral expressions for $\langle t\rangle$ and $\left\langle t^{2}\right\rangle$, we can obtain the numerical relationship between the mean first passage time and the ensemble temperature as well as the dependence relation between the relative fluctuation and the ensemble temperature.

The numerical results of the mean first passage time $\langle t\rangle$ and the relative fluctuation for the transition process from the stable wormhole phase to the unstable wormhole phase are presented in figure 8 . We are mainly interested in their temperature dependence. The temperature region is taken to be $\left[T_{1}, T_{2}\right]$, in which the double well shape of the free energy landscape emerges. It is shown that the mean first passage time for the transition process from the stable wormhole phase to the unstable wormhole phase is a monotonic 


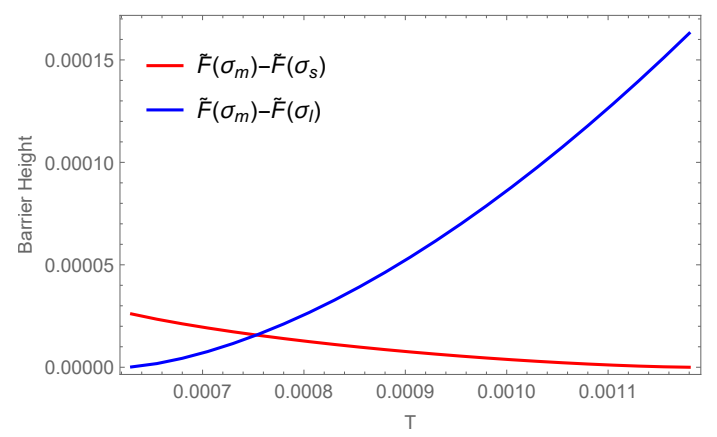

Figure 9. Barrier height (red line) between the stable wormhole phase (the green dot on the free energy landscape in figure 5) and the unstable wormhole phase (black dot on the free energy landscape) and barrier height (blue line) between the two black hole phase (the red dot on the free energy landscape in figure 5) and the unstable wormhole phase (black dot on the free energy landscape).

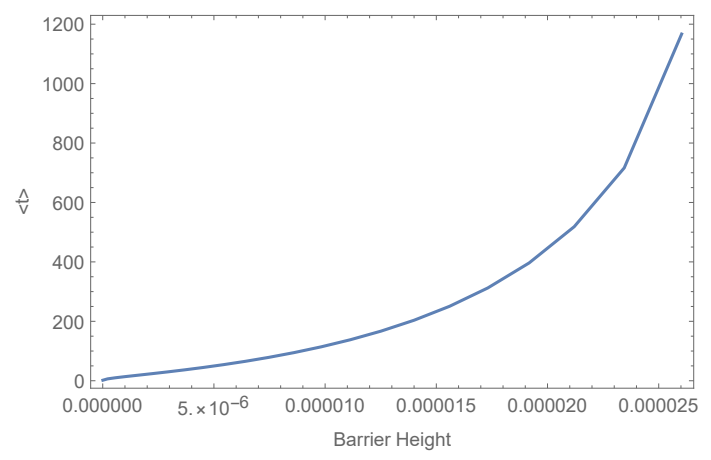

Figure 10. The correlation between the mean kinetic time $\langle t\rangle$ and the barrier height for the phase transition process from the stable wormhole phase to the unstable wormhole phase.

decreasing function of temperature. The main reason of this behavior is that the kinetics is determined by the barrier height between the two phases on the free energy landscape. When increasing the temperature, the barrier height on the free energy landscape between the stable wormhole phase and the unstable wormhole phase becomes smaller as shown in figure 9. In order to make this argument more clear, we have illustrated the correlation between the barrier height and the mean kinetic time $\langle t\rangle$ in figure 10. It is shown that kinetic time is a monotonous function of the barrier height. This illustrates how the free energy landscape topography in terms of the barrier height determines the kinetics of the phase transition. The second reason of the behavior of the kinetic time is due to the temperature, since the switching, which is the diffusion process caused by the thermal fluctuation, becomes more and more effective at higher temperatures.

The numerical results of the relative fluctuation of the kinetic time shows that the relative fluctuation is small in most of the temperature range while it becomes big when temperature approaches $T_{2}$. It is obvious that the relative fluctuations attain the maximum at the temperature where the mean first time is at its minimum. We believe that the behavior of the relative fluctuations of the first passage time is the consequence of the two 

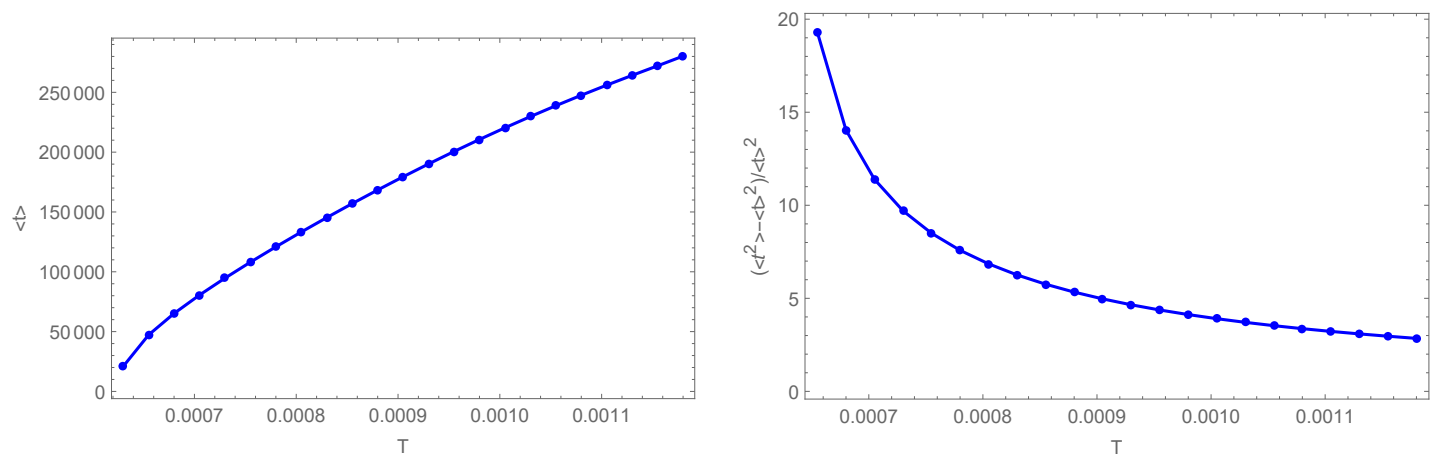

Figure 11. Mean first passage time (left panel) and the relative fluctuation (right panel) of the transition process from the two black hole phase to the unstable wormhole phase. In this plot, $q=50, \mathcal{J}=1$, and $\hat{\mu}=0.1$.

elements mentioned above. As the temperature increases, the free energy barrier height becomes smaller as shown in figure 9 while the temperature becomes higher. The higher thermal fluctuations relative to the barrier height indicate that the thermal fluctuations will have more significant impacts on the kinetics and associated fluctuations than the free energy barrier height at high temperatures. Therefore, the larger thermal fluctuations relative to the free energy barrier height lead to larger relative fluctuations in kinetics at high temperatures. At last, it can be concluded that the effect of the temperature dominates.

Now, we consider the kinetics and its fluctuation of the transition process from the two black hole phase (the red dot on the free energy landscape in figure 5) to the unstable wormhole phase (black dot on the free energy landscape). We also assume that this switching dominates the phase transition process from the two black hole phase to the stale wormhole phase. The numerical results of the mean first passage time $\langle t\rangle$ and the relative fluctuation for the transition process from the two black hole phase to the unstable wormhole phase are presented in figure 11. In this case, the mean first passage time is a monotonic increasing function of the ensemble temperature. At high temperature, the mean fist passage time becomes very long. This implies that it is very difficult for the two black hole phase to escape to the stable wormhole phase. The reason that the kinetic time becomes longer at higher temperature is that the barrier height on the free energy landscape between the two black hole phase and the unstable wormhole phase increases monotonically with the ensemble temperature as shown in figure 9 . In figure 12, we plot the correlation between the barrier height and the mean kinetic time $\langle t\rangle$ for the phase transition from the two black hole phase to the unstable wormhole phase. It is also shown that kinetic time is the monotonous function of the barrier height. Therefore, we can conclude that the free energy barrier is the dominate element that impacts the kinetic time of the transition process.

The behavior of the relative fluctuation as the function of the ensemble temperature is plotted on the right panel of figure 11. As discussed above, the relative fluctuation attains the maximum at the temperature where the mean first time is at its minimum. When 


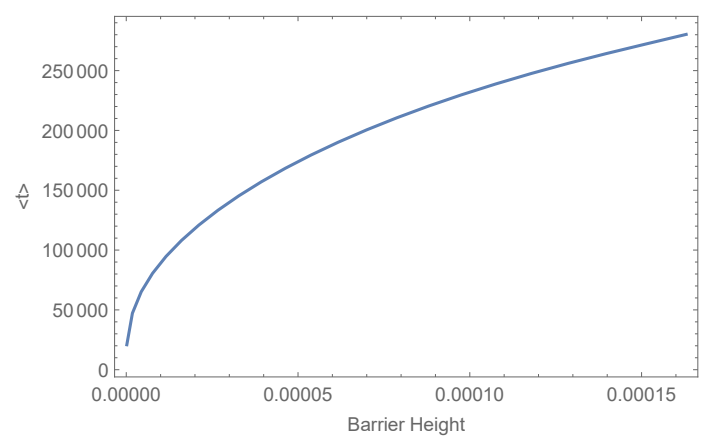

Figure 12. The correlation between the mean kinetic time $\langle t\rangle$ and the barrier height for the phase transition process from the two black hole phase to the unstable wormhole phase.

the ensemble temperature approaches $T_{1}$, the kinetic time $\langle t\rangle$ attains its minimum, while the relative fluctuation attains its maximum. As the temperature increases, the relative fluctuation decreases from the maximum to the minimum. It should be pointed out that, because of the considerable numerical errors for the kinetic time $\langle t\rangle$ and $\left\langle t^{2}\right\rangle$, it is difficult to compute the relative fluctuation precisely. However, the general trend is not affected by the numerical errors.

\section{Conclusion}

In the present work, we apply the stochastic dynamics method to study the phase transition in two coupled SYK models. For the two coupled SYK model at the large $N$ limit, according to the thermodynamics of the system, we define the off-shell free energy and quantify the corresponding free energy landscape. In the framework of the free energy landscape, we analyze the $\mu-T$ phase diagram and obtain the conclusion that the phase transition between the wormhole and two black hole in this model is analogous to the Van der Waals type, not exactly the Hawking-Page phase transition. Furthermore, we propose that the kinetics of the phase transition between the wormhole and two black hole can be investigated by the stochastic dynamics on the underlying free energy landscape. We assume the phase transition process between the wormhole and two black hole is a stochastic process under the thermal fluctuations and study the probabilistic Fokker-Planck equation which describes the kinetics of the phase transition process. By calculating the kinetics time and its fluctuation, we reveal the underlying thermodynamics and kinetics of the phase transition between the wormhole and two black hole. It is shown that the underlying reason of the kinetics behavior is determined by the barrier heights and the temperature on the free energy landscape.

Open Access. This article is distributed under the terms of the Creative Commons Attribution License (CC-BY 4.0), which permits any use, distribution and reproduction in any medium, provided the original author(s) and source are credited. 


\section{References}

[1] A. Einstein and N. Rosen, The Particle Problem in the General Theory of Relativity, Phys. Rev. 48 (1935) 73 [INSPIRE].

[2] R.W. Fuller and J.A. Wheeler, Causality and Multiply Connected Space-Time, Phys. Rev. 128 (1962) 919 [INSPIRE].

[3] M.S. Morris, K.S. Thorne and U. Yurtsever, Wormholes, Time Machines, and the Weak Energy Condition, Phys. Rev. Lett. 61 (1988) 1446 [INSPIRE].

[4] M. Visser, Lorentzian wormholes: From Einstein to Hawking, AIP, New York NY U.S.A. (1996).

[5] J. Maldacena and L. Susskind, Cool horizons for entangled black holes, Fortsch. Phys. 61 (2013) 781 [arXiv:1306.0533] [INSPIRE].

[6] P. Gao, D.L. Jafferis and A.C. Wall, Traversable Wormholes via a Double Trace Deformation, JHEP 12 (2017) 151 [arXiv:1608.05687] [INSPIRE].

[7] P. Hayden and J. Preskill, Black holes as mirrors: Quantum information in random subsystems, JHEP 09 (2007) 120 [arXiv:0708.4025] [INSPIRE].

[8] J. Maldacena, D. Stanford and Z. Yang, Diving into traversable wormholes, Fortsch. Phys. 65 (2017) 1700034 [arXiv: 1704.05333] [INSPIRE].

[9] A. Almheiri, T. Hartman, J. Maldacena, E. Shaghoulian and A. Tajdini, The entropy of Hawking radiation, Rev. Mod. Phys. 93 (2021) 035002 [arXiv:2006.06872] [INSPIRE].

[10] G. Penington, S.H. Shenker, D. Stanford and Z. Yang, Replica wormholes and the black hole interior, arXiv:1911.11977 [INSPIRE].

[11] A. Almheiri, T. Hartman, J. Maldacena, E. Shaghoulian and A. Tajdini, Replica Wormholes and the Entropy of Hawking Radiation, JHEP 05 (2020) 013 [arXiv:1911.12333] [INSPIRE].

[12] J.L. Blázquez-Salcedo, C. Knoll and E. Radu, Traversable wormholes in Einstein-Dirac-Maxwell theory, Phys. Rev. Lett. 126 (2021) 101102 [arXiv:2010.07317] [INSPIRE].

[13] J. Maldacena and A. Milekhin, Humanly traversable wormholes, Phys. Rev. D 103 (2021) 066007 [arXiv:2008.06618] [INSPIRE].

[14] J. Maldacena and X.-L. Qi, Eternal traversable wormhole, arXiv:1804.00491 [INSPIRE].

[15] A. Almheiri and J. Polchinski, Models of AdS $S_{2}$ backreaction and holography, JHEP 11 (2015) 014 [arXiv: 1402.6334] [INSPIRE].

[16] K. Jensen, Chaos in AdS $S_{2}$ Holography, Phys. Rev. Lett. 117 (2016) 111601 [arXiv: 1605.06098] [INSPIRE].

[17] J. Maldacena, D. Stanford and Z. Yang, Conformal symmetry and its breaking in two dimensional Nearly Anti-de-Sitter space, Prog. Theor. Exp. Phys. 2016 (2016) 12C104 [arXiv: 1606.01857] [INSPIRE].

[18] J. Engelsöy, T.G. Mertens and H. Verlinde, An investigation of $A d S_{2}$ backreaction and holography, JHEP 07 (2016) 139 [arXiv: 1606.03438] [INSPIRE].

[19] C. Teitelboim, Gravitation and Hamiltonian Structure in Two Space-Time Dimensions, Phys. Lett. B 126 (1983) 41 [inSPIRE]. 
[20] R. Jackiw, Lower Dimensional Gravity, Nucl. Phys. B 252 (1985) 343 [InSPIRE].

[21] S.W. Hawking and D.N. Page, Thermodynamics of Black Holes in anti-de Sitter Space, Commun. Math. Phys. 87 (1983) 577 [InSPIRE].

[22] S. Sachdev and J. Ye, Gapless spin fluid ground state in a random, quantum Heisenberg magnet, Phys. Rev. Lett. 70 (1993) 3339 [cond-mat/9212030] [INSPIRE].

[23] A. Kitaev, A simple model of quantum holography, talk given at the Entanglement in Strongly-Correlated Quantum Matter, KITP, Santa Barbara, CA, U.S.A., 6 April-2 July 2015.

[24] A. Kitaev and S.J. Suh, The soft mode in the Sachdev-Ye-Kitaev model and its gravity dual, JHEP 05 (2018) 183 [arXiv:1711.08467] [INSPIRE].

[25] J. Maldacena and D. Stanford, Remarks on the Sachdev-Ye-Kitaev model, Phys. Rev. D 94 (2016) 106002 [arXiv:1604.07818] [INSPIRE].

[26] G.T. Horowitz, D. Marolf, J.E. Santos and D. Wang, Creating a Traversable Wormhole, Class. Quant. Grav. 36 (2019) 205011 [arXiv:1904.02187] [INSPIRE].

[27] X.-L. Qi and P. Zhang, The Coupled SYK model at Finite Temperature, JHEP 05 (2020) 129 [arXiv: 2003.03916] [INSPIRE].

[28] P. Zhang, More on Complex Sachdev-Ye-Kitaev Eternal Wormholes, JHEP 03 (2021) 087 [arXiv:2011.10360] [INSPIRE].

[29] N. Sorokhaibam, Phase transition and chaos in charged SYK model, JHEP 07 (2020) 055 [arXiv: 1912.04326] [INSPIRE].

[30] A.M. García-García and V. Godet, Euclidean wormhole in the Sachdev-Ye-Kitaev model, Phys. Rev. D 103 (2021) 046014 [arXiv:2010.11633] [InSPIRE].

[31] J. Maldacena and A. Milekhin, SYK wormhole formation in real time, JHEP 04 (2021) 258 [arXiv: 1912.03276] [INSPIRE].

[32] H. Frauenfelder, S.G. Sligar and P.G. Wolynes, The energy landscapes and motions of proteins, Science 254 (1991) 1598.

[33] H. Frauenfelder and P.G. Wolynes, Biomolecules: Where the physics of complexity and simplicity meet, Phys. Today 47 (1994) 58.

[34] N. Goldenfeld, Lectures On Phase Transitions And The Renormalization Group, Westview Press (1992).

[35] J. Wang, Landscape and flux theory of non-equilibrium dynamical systems with application to biology, Adv. Phys. 64 (2015) 1.

[36] D.C. Johnston, Thermodynamic Properties of the van der Waals Fluid, arXiv:1402.1205.

[37] D. Kubiznak and R.B. Mann, P - V criticality of charged AdS black holes, JHEP 07 (2012) 033 [arXiv: 1205.0559] [INSPIRE].

[38] R. Li, K. Zhang and J. Wang, Thermal dynamic phase transition of Reissner-Nordström Anti-de Sitter black holes on free energy landscape, JHEP 10 (2020) 090 [arXiv: 2008. 00495] [INSPIRE].

[39] R. Li and J. Wang, Thermodynamics and kinetics of Hawking-Page phase transition, Phys. Rev. D 102 (2020) 024085 [INSPIRE]. 
[40] R. Li, K. Zhang and J. Wang, Probing black hole microstructure with the kinetic turnover of phase transition, Phys. Rev. D 104 (2021) 084076 [arXiv: 2102.09439] [INSPIRE].

[41] S.-W. Wei, Y.-X. Liu and Y.-Q. Wang, Dynamic properties of thermodynamic phase transition for five-dimensional neutral Gauss-Bonnet AdS black hole on free energy landscape, arXiv:2009.05215 [INSPIRE].

[42] R. Li and J. Wang, Energy and entropy compensation, phase transition and kinetics of four dimensional charged Gauss-Bonnet Anti-de Sitter black holes on the underlying free energy landscape, arXiv:2012.05424 [INSPIRE].

[43] S.-W. Wei, Y.-Q. Wang, Y.-X. Liu and R.B. Mann, Observing dynamic oscillatory behavior of triple points among black hole thermodynamic phase transitions, Sci. China Phys. Mech. Astron. 64 (2021) 270411 [arXiv:2102.00799] [INSPIRE].

[44] R.G. Cai, Oscillatory behaviors near a black hole triple point, Sci. China Phys. Mech. Astron. 64 (2021) 290432.

[45] S.-Q. Lan, J.-X. Mo, G.-Q. Li and X.-B. Xu, Effects of dark energy on dynamic phase transition of charged AdS black holes, Phys. Rev. D 104 (2021) 104032 [arXiv:2104.11553] [INSPIRE].

[46] R. Li, K. Zhang and J. Wang, Kinetics and its turnover of Hawking-Page phase transition under the black hole evaporation, Phys. Rev. D 104 (2021) 084060 [arXiv:2105.00229] [INSPIRE].

[47] S.-J. Yang, R. Zhou, S.-W. Wei and Y.-X. Liu, Dynamics and kinetics of phase transition for Kerr AdS black hole on free energy landscape, arXiv:2105.00491 [INSPIRE].

[48] J.-X. Mo and S.-Q. Lan, Dynamic phase transition of charged dilaton black holes, arXiv:2105.00868 [INSPIRE].

[49] A.N. Kumara, S. Punacha, K. Hegde, C.L.A. Rizwan, K.M. Ajith and M.S. Ali, Dynamics and kinetics of phase transition for regular AdS black holes in general relativity coupled to non-linear electrodynamics, arXiv:2106.11095 [INSPIRE].

[50] S.-W. Wei and Y.-X. Liu, Insight into the Microscopic Structure of an AdS Black Hole from a Thermodynamical Phase Transition, Phys. Rev. Lett. 115 (2015) 111302 [Erratum ibid. 116 (2016) 169903] [arXiv: 1502.00386] [INSPIRE].

[51] J.W. York Jr., Black hole thermodynamics and the Euclidean Einstein action, Phys. Rev. D 33 (1986) 2092 [INSPIRE].

[52] B.F. Whiting and J.W. York Jr., Action Principle and Partition Function for the Gravitational Field in Black Hole Topologies, Phys. Rev. Lett. 61 (1988) 1336 [INSPIRE].

[53] R. André and J.P.S. Lemos, Thermodynamics of five-dimensional Schwarzschild black holes in the canonical ensemble, Phys. Rev. D 102 (2020) 024006 [arXiv:2006.10050] [INSPIRE].

[54] R. André and J.P.S. Lemos, Thermodynamics of d-dimensional Schwarzschild black holes in the canonical ensemble, Phys. Rev. D 103 (2021) 064069 [arXiv:2101.11010] [INSPIRE].

[55] C. Liu and J. Wang, Path integral and instantons for the process and phase transition rate of the RNAdS black hole, arXiv:2109.14319 [INSPIRE]. 